\title{
Foramen Magnum Meningioma and Arachnoid Cyst Coinciding in the Lateral Cerebellomedullary Cistern
}

\author{
- Case Report-
}

\author{
Kiyoyuki YANAKa, Akio Hyodo, Hiroyuki FujImoRi, Yoshihiko Yoshi \\ and Tadao NOSE
}

\author{
Department of Neurosurgery, Institute of Clinical Medicine, University of Tsukuba, \\ Tsukuba, Ibaraki
}

\begin{abstract}
An extremely rare foramen magnum meningioma associated with an arachnoid cyst in the lateral cerebellomedullary cistern occurred in a 65 -year-old female presenting with dizziness. Neuroimaging revealed a meningioma at the left lateral edge of the foramen magnum and an arachnoid cyst mainly located in the right lateral cerebellomedullary cistern, compressing the medulla oblongata bilaterally. After fenestration of the cyst wall and tumor removal, the clinical symptoms ameliorated. We recommend that where a foramen magnum tumor coexists with an arachnoid cyst of the posterior fossa, the tumor should be removed after shrinking the cyst to obviate the need for brainstem retraction.
\end{abstract}

Key words: arachnoid cyst, cerebellomedullary cistern, foramen magnum, meningioma, posterior fossa

\section{Introduction}

Foramen magnum meningiomas are uncommon, representing about $3.2 \%$ of all intracranial meningiomas. ${ }^{6}$ In contrast, $23 \%$ of all arachnoid cysts occur in the posterior fossa, the second most common location. ${ }^{4)}$ However, arachnoid cysts in the cerebellomedullary cistern are extremely rare. ${ }^{57}$ Here, we report the association of a foramen magnum meningioma and an arachnoid cyst in the cerebellomedullary cistern, and discuss the clinical and therapeutic features of this condition.

\section{Case Report}

A 65-year-old female with a 20 -year history of dizziness and recent nuchal pain was admitted because of recently worsened symptoms. On admission, she was alert and oriented, with intact cognitive functions. Neurological examination revealed horizontal nystagmus and hyper-reflexia without motor paresis. Other signs related to lower cranial nerves were nor-

Received October 1, 1991; Accepted December 27 , 1991 mal. The otolaryngological examination was also normal.

Plain skull x-ray films showed no abnormality. Precontrast computed tomographic (CT) scans revealed a small isodense area at the left lateral edge of the foramen magnum, and a low-density area in the right lateral cerebellomedullary cistern compressing the medulla oblongata bilaterally (Fig. 1 left). The isodense mass was enhanced postcontrast (Fig. 1 $r i g h t$ ). Magnetic resonance (MR) imaging showed the mass on the left side as isointense on the $T_{1}$-weighted image and hyperintense on the $T_{2}$-weighted image, enhanced after intravenous administration of gadolinium-diethylenetriaminepenta-acetic acid (GdDTPA) (Fig. 2). The mass on the right side appeared as a homogeneous signal consistent with a cerebrospinal fluid-containing cyst, extending from the cerebellopontine angle to the lateral cerebellomedullary cistern, but mainly located in the latter. CT cisternograms revealed a cystic mass with delayed filling of contrast medium in the corresponding area (Fig. 3). Digital subtraction angiograms (DSAs) revealed that the mass on the left was fed by a branch of the ascending pharyngeal artery (Fig. 4). The mass on the left was therefore diagnosed as 

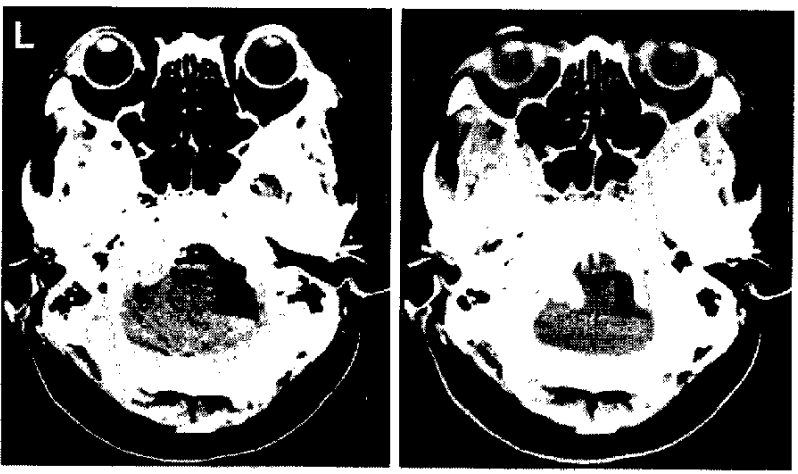

Fig. 1 left: Precontrast CT scan, showing an isodense mass at the left lateral edge of the foramen magnum and a low-density mass in the right lateral cerebellomedullary cistern. right: Postcontrast CT scan, showing enhancement of the mass on the left.

a meningioma at the foramen magnum and that on the right as an arachnoid cyst in the cerebellomedullary cistern.

The tumor feeding artery was superselectively embolized to reduce intraoperative bleeding. Fenestration of the cyst wall and tumor removal were accomplished via the posterior approach, with suboccipital craniectomy and C1 laminectomy. The cyst with a thick membrane wall was exposed and the cyst wall widely fenestrated. The round, soft, and slightly pinkish tumor was then totally removed requiring little retraction of the brainstem. Histological examination of samples indicated meningothelial meningioma and arachnoid membrane (Fig. 5).

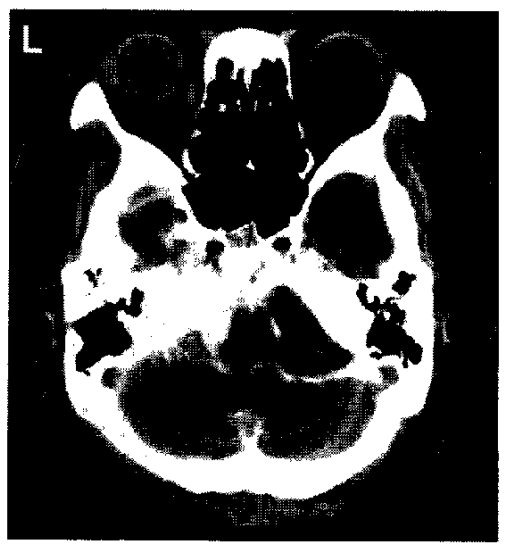

Fig. 3 CT cisternogram, showing a cystic lesion with delayed filling of contrast medium in the right lateral cerebellomedullary cistern.

Postoperative CT scans revealed that the tumor had been removed completely and the cyst size had decreased. The postoperative course was uneventful and her dizziness and nuchal pain improved.

\section{Discussion}

The most frequent symptoms of foramen magnum meningiomas are dysesthesia of the fingers and pain in the suboccipital region. Other symptoms are gait disturbance, incoordination or clumsiness of the hands, and weakness of the extremities. ${ }^{6}$ Dizziness is infrequent, occurring in only $1.8-3.9 \%$ of cases reported. ${ }^{2,6)}$ Therefore, there is no pathognomonic symptom for this condition.
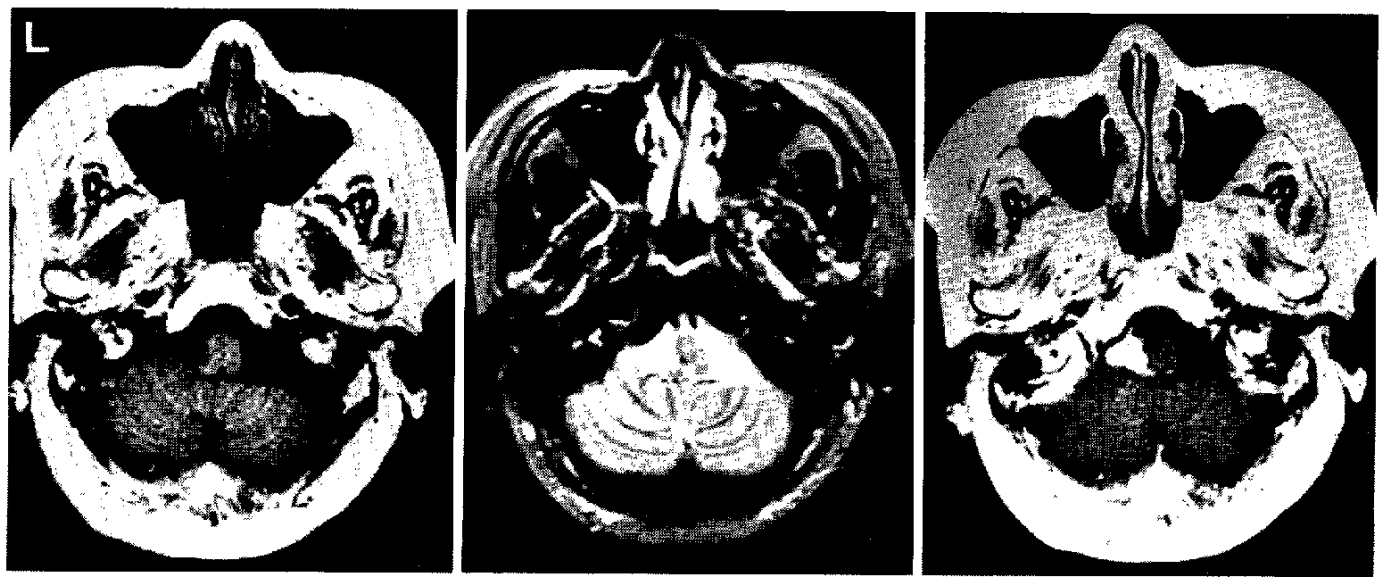

Fig. 2 T $\mathrm{T}_{1}$-weighted (left; SE 340/11 msec), $\mathrm{T}_{2}$-weighted (center; SE $3000 / 90 \mathrm{msec}$ ), and Gd-DTPAenhanced (right; SE $340 / 11 \mathrm{msec}$ ) MR images, showing a small solid mass on the left and a cystic mass on the right. The medulla oblongata is compressed bilaterally. 


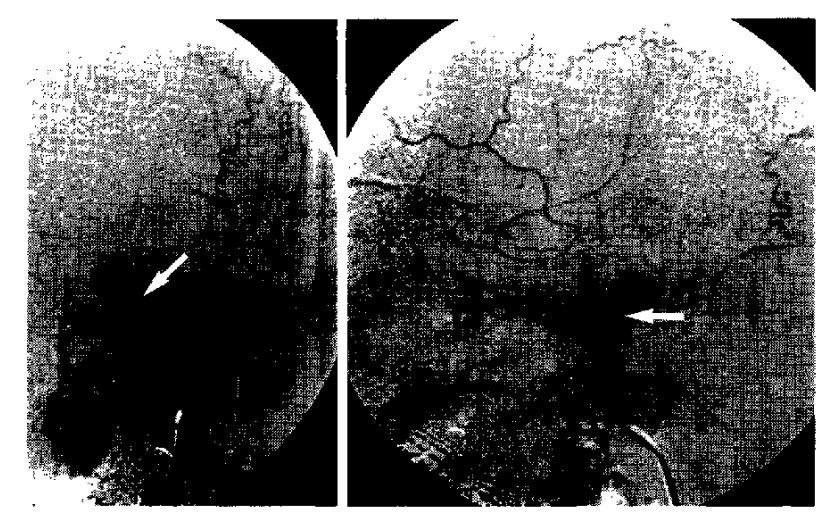

Fig. 4 DSAs of the left external carotid artery, anteroposterior (left) and lateral (right) views, showing the ascending pharyngeal artery feeder (arrow) and tumor staining.

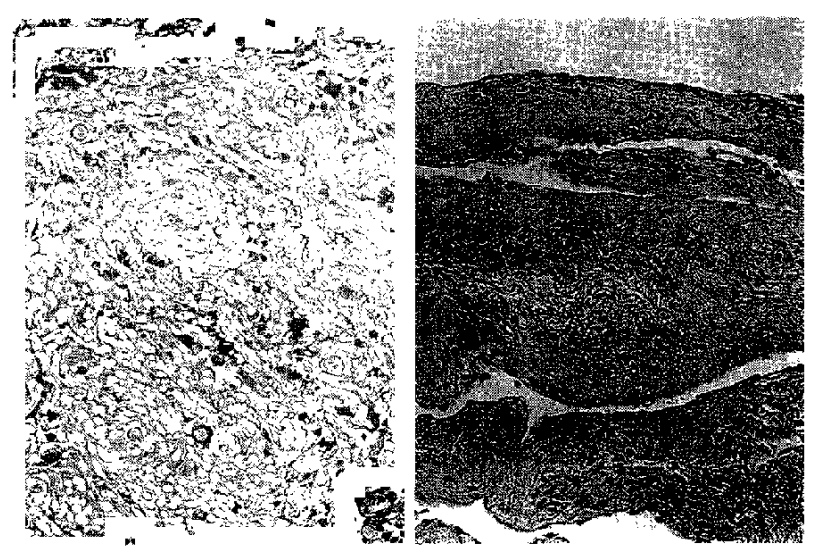

Fig. 5 Photomicrographs of the operative specimen, showing meningioma cells with indistinct cytoplasmic margins and uniform round or oval nuclei (left), and arachnoid membrane (right). HE stain, $\times 125$.

In contrast, the clinical syndrome caused by an arachnoid cyst in the cerebellopontine angle may closely mimic that of acoustic neurinoma. ${ }^{3)}$ Only one case of arachnoid cyst of the cerebellomedullary cistern presenting with lower cranial nerve palsy has been reported. ${ }^{5}$ Therefore, such an arachnoid cyst may cause dizziness and/or lower cranial nerve paresis by involving the cranial nerves or compressing the brain parenchyma.

In our case, the medulla oblongata was compressed bilaterally by the arachnoid cyst and the meningioma. The cerebellum was also compressed by the arachnoid cyst. The long history of dizziness may have resulted from compression of the medulla oblongata and the cerebellum by the arachnoid cyst, with growth of the meningioma causing the recent deterioration in symptom.

An enlarged cistern of the posterior fossa associated with neurinomas and meningiomas frequently occurs. However, in our case CT cisternography revealed cyst formation with delayed filling of contrast medium, suggesting that the lesion was not an enlarged cistern.

The association of meningiomas with other intracranial mass lesions is partly coincidental, but meningiomas and other lesions do occur in the same area, which may suggest a common etiology." There was no clear mechanism causing both the arachnoid cyst and meningioma of the posterior fossa in our case. However, the cyst wall was thicker than arachnoid membrane elsewhere. This may indicate an association between the cyst and meningioma.

In our case, microsurgical removal of the foramen magnum meningioma was performed after fenestrating the cyst wall, which greatly facilitated the procedure. We recommend that where a foramen magnum tumor coexists with an arachnoid cyst of the posterior fossa, the tumor should be removed after shrinking the cyst to obviate the need for brainstem retraction.

\section{Acknowledgment}

The authors are grateful to Dr. Masami Itoh of Department of Neurosurgery, Satte General Hospital for preparation of the manuscript and to Dr. Kiyoshi Matsueda of Department of Radiology, Institute of Clinical Medicine, University of Tsukuba for preparation of the photographs.

\section{References}

1) Bradac GB, Ferszt R, Kendall B: Cranial Meningiomas. Diagnosis-Biology-Therapy. New York, SpringerVerlag, 1990, pp 2-13

2) Meyer FB, Ebersold MJ, Reese DF: Benign tumors of the foramen magnum. $J$ Neurosurg $61: 136-142,1984$

3) Rengachary SS: Intracranial arachnoid and ependymal cysts, in Wilkins RH, Rengachary SS (eds): Neurosurgery III. New York, McGraw-Hill, 1985, pp 2160-2172

4) Rengachary SS, Watanabe I: Ultrastructure and pathogenesis of intracranial arachnoid cysts. $J$ Neuropathol Exp Neurol 40: 61-83, 1981

5) Takechi A, Uozumi T, Yamanaka M, Kanazawa J, Hatayama $T$, Sumida $M$, Kajima $T$ : A case of arachnoid cyst in the posterior fossa with lower cranial nerve palsy. No Shinkei Geka 18: 551-554, 1990 (in Japanese) 
6) Yasuoka S, Okazaki H, Daube JR, MacCarty CS: Foramen magnum tumors: Analysis of 57 cases of benign extramedullary tumors. $J$ Neurosurg 49: 828838,1978
Address reprint requests to: K. Yanaka, M.D., Department of Neurosurgery, Institute of Clinical Medicine, University of Tsukuba, 2-1-1 Amakubo, Tsukuba, Ibaraki 305, Japan. 